\title{
Gisela Harras
}

\section{Jenseits von semantischen Konventionen - zum Beispiel: tautologische Äußerungen}

1. Sprachliche Konventionen und kooperative Sprechersetzungen

2. Tautologische Äußerungen und ihre Interpretationsmöglichkeiten gegen die Logik

3. Semantische Erklärungen der Informativität tautologischer Äußerungen und deren Fragwürdigkeiten

4. Radikal pragmatische Lösungen

5. Ausblick

1. Sprachliche Konventionen und kooperative Sprechersetzungen

Es ist sicherlich das unschätzbare Verdienst des Sprachphilosophen Paul Grice, nicht nur auf den fundamentalen Unterschied zwischen Konventionalität der Sprache als repräsentationalem Kenntnissystem und Kooperativität von Sprechern als prozeduralem Kenntnissystem hingewiesen, sondern auch die systematischen Aspekte dieses Unterschieds in seinen Beiträgen zum Thema "Logic and Conversation“ (vgl. Grice, 1989) herausgearbeitet zu haben. Der interessanteste Punkt der Griceschen Überlegungen ist m. E. der folgende: Konventionen der Sprache legen Sprecher und Hörer gleichermaßen auf bestimmte Ausschnitte von Überzeugungssystemen fest; sie gelten nicht nur für einen jeweiligen Augenblick des Kommunizierens, sondern sind für jedes Mitglied der Gemeinschaft in jeder Situation verbindlich. Jemand, der den folgenden Satz äußert:

(1) Hans hat aufgehört zu rauchen

legt sich verbindlich auf den Sachverhalt: Hans hat bis zu einem bestimmten Zeitpunkt $t$ geraucht fest; er ist dazu konventionell verpflichtet, wenn er den Ausdruck aufhören verwendet. Jemand, der sagt:

(2) Alle Philosophen akzeptieren diese These

legt sich verbindlich auf den Sachverhalt: einige Philosophen akzeptieren diese These fest; durch die Verwendung des Quantors alle wird qua Konvention impliziert, daß einige Philosophen diese These akzeptieren. Wenn man sagt:

(3) Hansens Kinder haben Glatzen

dann gibt man mit dieser Äußerung auch zu verstehen, daß Hans Kinder hat; (15) könnte weder wahr noch falsch sein, wenn nicht zuträfe, daß Hans Kinder hat. Der Gebrauch der definiten Nominalphrase Hansens Kinder legt konventionell das Zutreffen der Präsupposition Hans hat Kinder fest. 
Konventionen konstituieren, wie es Lewis formuliert hat, eine Gruppe in ihrer Eigenschaft als Gruppe - ihre Mitglieder gleichen einander wie ein Ei dem anderen.

Kooperative Festlegungen hingegen eröffnen Spielräume, die situationsabhängig und individuell je nach Sprecherintentionen geschaffen werden können. Diese Freiräume können natürlich nur unter der Bedingung der wechselseitigen Kooperationsunterstellung der jeweiligen Kommunikationspartner genutzt werden. Unter dieser Voraussetzung werden primär nicht-konventionell geregelte Meinens- und Verstehensfundamente aufgebaut, die innerhalb einer Gruppe spezifische Kommunikationsgemeinschaften begründen können. Wenn jemand z. B. auf die Frage: „Wo ist Hans?“ antwortet:

(4) Vor der Post steht ein roter VW

dann kann er damit zu verstehen geben wollen, daß Hans in der Post ist. Dies ist nun nicht qua Konvention durch die sprachliche Äußerung impliziert; der Hörer kann diese Information nicht aus der sprachlichen Form allein erschlieBen, er braucht dazu eine Reihe von Annahmen und ein spezifisches Hintergrundswissen über die Äußerungssituation. Wie man sich solche Annahmen und das Format des Hintergrundswissens vorzustellen hat, ist bekanntlich durch die Gricesche Kommunikationstheorie präzisiert worden. Obwohl diese zu den obligaten Truismen linguistischen Allgemeinwissens gehört, sei hier nochmal - der Eindeutigkeit des Verweises halber - eine gängige Version derselben wiedergegeben.

Kommunikation zeichnet sich im wesentlichen durch Kooperation der jeweils daran Beteiligten aus. Als oberster Grundsatz gilt ein Kooperationsprinzip (KP), das Grice so formuliert:

Kooperationsprinzip (KP):

Mache deinen Gesprächsbeitrag jeweils so, wie es von dem akzeptierten Zweck oder der akzeptierten Richtung des Gesprächs, an dem du teilnimmst, gerade verlangt wird.

Dieses Prinzip wird durch vier Kriterien, die bei Grice Maximen heißen, näher bestimmt:

\section{Maxime der Quantität}

Mache deinen Beitrag so informativ wie für die gegebenen Zwecke nötig

Mache deinen Beitrag nicht informativer als nötig

2. Maxime der Qualität

Versuche deinen Beitrag so zu machen, daß er wahr ist

Sage nichts, was du für falsch hältst

Sage nichts, wofür dir angemessene Gründe fehlen

3. Maxime der Relevanz

Sei relevant! 
4. Maxime der Modalität (Art und Weise)

Sei klar!

Vermeide Dunkelheit des Ausdrucks

Vermeide Mehrdeutigkeiten

Sei kurz, vermeide unnötige Weitschweifigkeiten

Der Reihe nach, sei folgerichtig

Dieses Kooperationsprinzip und seine Maximen sind keine moralischen Forderungen an die Kommunikationsteilnehmer; sie beschreiben auch nicht die alltägliche Praxis des Kommunizierens, sondern sie sind Unterstellungen, die die Kommunikationspartner wechselseitig machen. Kommunizieren heißt, davon ausgehen, daß das Prinzip und die Maximen im allgemeinen eingehalten werden. Sie müssen dies allerdings nicht immer, sie können sogar absichtlich verletzt oder ausgebeutet werden, und diese Erkenntnis führt hörerseitig zu einer sinnvollen Interpretation einer Sprecheräußerung. Ein Beispiel für eine solche Inferenz ist folgendes: jemand äußert:

(5) Kinder sind Kinder

Der Hörer erkennt, daß der Sprecher gegen die Maxime der Informativität verstößt, geht aber davon aus, daß sich der Sprecher kooperativ verhält, $d . h$. ihm etwas bestimmtes zu verstehen geben will - also zieht er eine informative Inferenz derart: das ist die Art des undisziplinierten Verhaltens, das man von Kindern erwarten muß. Ob diese Wiedergabe der inferierten Information allerdings korrekt ist, werden wir noch sehen.

2. Tautologische Äußerungen und ihre Interpretationsmöglichkeiten gegen die Logik

Als prototypische Beispiele für tautologische Äußerungen seien hier zunächst die folgenden betrachtet:

(6) Krieg ist Krieg

(7) Kinder sind Kinder

(8) Die Ehre ist die Ehre

(9) Ein Junggeselle ist ein Junggeselle usw.

Urteile der Form „a ist a“ werden aus der Sicht der formalen Logik als uninformativ, aber sinnvoll angesehen. Sinnvoll sind sie deshalb, weil sie notwendigerweise immer wahr sind und weil sie die Relation ausdrücken, in der ein Gegenstand allein zu sich selbst und zu keinem anderen Gegenstand steht (vgl. z. B. Tugendhat, 1976; Tugendhat/Wolf, 1983). Gegen die Auffassung der Uninformativität kann man nun mit Blick auf unsere natürliche Sprache eine ganze Reihe von Einwänden haben. Einen der besten liefert bereits Lessing: In seiner Komödie „Minna von Barnhelm“ wehrt der starrsinnige Major von Tellheim alle Hilfe Minnas ab, und der Dialog der beiden endet in einer Tautologie: 
Tellheim: Ich brauche keine Gnade, ich will Gerechtigkeit, meine Ehre ...

Minna: Die Ehre eines Mannes wie Sie ...

Tellheim (hitzig): Nein, mein Fräulein, Sie werden von allen Dingen recht gut urteilen können, nur hierüber nicht. Die Ehre ist nicht die Stimme unseres Gewissens, nicht das Zeugnis weniger Rechtschaffenen ...

Minna: Nein, nein, ich weiß wohl - die Ehre ist - die Ehre

Man wird kaum behaupten wollen, daß Minnas Äußerung in dem wiedergegebenen Zusammenhang uninformativ sei.

Es gibt nun zunächst drei formal-semantische Eigenschaften tautologischer Äußerungen, die im Widerspruch zu ihren logischen Eigenschaften der Uninformativität und der notwendigen Wahrheit stehen:

(1) aus der Uninformativität müßte folgen, daß man einen der beiden nominalen Ausdrücke durch einen synonymen ersetzen könnte, ohne den Wahrheitswert des gesamten Satzes zu ändern. Wenn man nun aber in einer Äußerung wie:

(10) Ein Krankenhaus ist ein Krankenhaus

den Ausdruck Krankenhaus in der ersten Nominalphrase durch das Synonym Hospital ersetzen würde:

$\left(10^{\prime}\right)$ Ein Hospital ist ein Krankenhaus

würde die Äußerung ihre Tautologiehaftigkeit verlieren und statt dessen eher als eine Art Worterklärung interpretiert werden wie auch in dem folgenden Beispiel:

(11) beinah ist beinah

(11') beinah ist fast

(2) Wenn tautologische Äußerungen zugleich uninformativ und notwendig wahr sind, müßten alle tautologischen Äußerungen synonym zueinander sein, d. h. sie müßten zumindest in einigen Kontexten austauschbar sein. Es gibt nun aber keinen denkbaren Kontext, in dem man die Aussage

(12) Krieg ist Krieg •

durch die Aussage

(13) Paris ist Paris

salva veritate ersetzen könnte, d.h. die logische Position führt für die natürliche Sprache in eine Absurdität.

(3) Tautologische Äußerungen sind der Logik zufolge notwendig wahr, nur Toren oder Menschen, die der Sprache oder ihrer Sinne nicht mächtig sind, würden sie bestreiten. Nun kommen in der natürlichen Sprache auch Äußerungen wie die folgenden vor:

(14) Heute bin ich nicht ich

(15) Rom ist nicht mehr Rom

(16) Mein Vater ist nicht mein Vater

(17) Wein ist nicht Wein

usw. 
Der Logik zum Trotz können wir uns mühelos Situationen ausdenken, in denen wir mit tautologischen Äußerungen etwas Informatives meinen, ein paar Beispiele:

(18) Ich bin ich

könnte in einer Situation geäußert werden, in der der Vater seinen Sohn schon wieder mal gerügt hat, daß er nur eine 3 in Mathematik geschrieben hat. Darauf entgegnet der Sohn entnervt Satz (18).

(19) Rom ist Rom

Herrn Maier ist auch bei seinem vierten Rombesuch wieder das Auto aufgebrochen worden. Daraufhin seufzt er resignierend Satz (19).

(20) Hans ist Hans

Der ungeschickte Hans hat mal wieder sein Bierglas umgeworfen, und die Geschädigten fügen sich in ihr Schicksal mit Satz (20).

(21) Mein Vater ist mein Vater

Karin ärgert sich schon lange darüber, da $\beta$ ihr Freund Hans seinem Vater nicht nur immer aufs Wort folgt, sondern auch dessen sämtliche Lebensweisheiten übernimmt. Endlich faßt sie sich ein Herz und fordert Hans auf, auch einmal eine Entscheidung zu treffen, notfalls gegen den Vater. Woraufhin Hans ganz entsetzt (21) äußert.

Dies sind Kontexte oder Situationen, in denen solche Äußerungen gemacht werden können; ihre Umstände sind allerdings nicht die Bedingungen des korrekten Gebrauchs der Äußerungen: der Sohn, dem die schlechte Note vorgeworfen wird, hätte auch nur niedergeschmettert dreinschauen können, oder der Vater hätte seinen Sohn dafür loben können, schon wieder eine 3 geschrieben zu haben, worauf der Sohn mit stolzgeschwellter Brust (18) äußert.

Bei soviel Kontextabhängigkeit bleibt für die natürliche Sprache die Frage nach der Bedeutung, d. h. der semantischen Information solcher Äußerungen. Kann man überhaupt von einer solchen reden oder haben sie nur einen kommunikativen Sinn, d. h. eine bestimmte Funktion in der Kommunikation, und wenn letzteres, welche, und wie soll ein Hörer diese jeweilige Funktion erschließen? Anders gefragt: ist die Erklärung der Informativität von tautologischen Äußerungen eine Sache der Semantik einer jeweiligen natürlichen Sprache oder ist sie eine Sache der universellen Pragmatik des Kommunizierens? Ich werde im Folgenden zunächst auf zwei Möglichkeiten der semantischen Erklärung und dann auf pragmatische Lösungen eingehen.

\section{Semantische Erklärungen der Informativität tautologischer Äußerungen und deren Fragwürdigkeiten}

Soweit ich sehe, gibt es zwei Möglichkeiten der semantischen Erklärung: eine, die sich an der Semantik von Identitätssätzen allgemein orientieren könnte, und eine, die von Wierzbicka stammt und auf durch tautologische Äußerungen ausgedrückte Einstellungen rekurriert. 
Zur ersten Möglichkeit: Identitätssätze drücken die Identität eines Gegenstands mit ihm selbst so aus, daß der Gegenstand durch zwei singuläre Terme bezeichnet wird, die ihn durch verschiedene Gegebenheitsweisen identifizieren (vgl. Tugendhat/Wolf, 1983). Ein berühmtes Beispiel:

(22) Der Abendstern ist der Morgenstern

Der Gegenstand wird hier durch die beiden Gegebenheiten „Abendstern“ und „Morgenstern“ identifiziert. Die beiden Ausdrücke haben den gleichen Referenten, aber einen jeweils unterschiedlichen Sinn, d. h. der identische Referent wird durch unterschiedliche Arten seines Gegebenseins charakterisiert.

Man könnte nun Identitätssätze der Form $\mathrm{a}=\mathrm{a}$ als einen Spezialfall von Identitätssätzen betrachten, mit der Spezifikation, daß der Referent zweimal durch den gleichen Ausdruck identifiziert wird, wobei jeweils der gleiche Ausdruck einen anderen Aspekt oder eine andere Gegebenheitsweise des Referenten wiedergibt. Die Gegebenheitsweise des ersten Ausdrucks ist global, unspezifisch, die des zweiten spezifischer. Ein Muster für eine Paraphrase wäre folgendes:

(23) Das, worauf der Ausdruck „a“" referiert, ist das (proto)typische A

Eine Äußerung wie: (5) „Krieg ist Krieg“ wäre dann zu paraphrasieren als:

(24) Das worauf der Ausdruck Krieg referiert, ist der (proto)typische Krieg

Aus der sprachlich determinierten Kenntnis der Wörter würde der Interpret unter geeigneten Umständen dann zu sowas kommen wie: Krieg ist ein kollektives, naturkatastrophenartiges, schreckliches, leidbringendes Ereignis.

Dieser Lösungsversuch auf der Ebene der Semantik birgt nun allerdings einige Rätsel, z. B.:

- wieso kann man davon ausgehen, daß der Ausdruck Krieg in unterschiedlicher Bedeutung verwendet ist?

- Prototypen sind kontextabhängig, wie die psychologischen Experimente gezeigt haben; welche Kontexte sind für welche Ausprägungen von Prototypen verantwortlich?

- wie läßt sich von der hier vorgeschlagenen Interpretation von tautologischen Äußerungen auf mögliche Funktionen in der Kommunikation schließen?

Ein anderer semantischer Lösungsvorschlag für tautologische Äußerungen stammt von Anna Wierzbicka $(1987 ; 1988)$, die für eine radikale Semantik plädiert. Ihrer Meinung nach ist die Interpretation von tautologischen Äußerungen sprachspezifisch. Als Beweis dafür führt sie verschiedene Formulierungsmöglichkeiten von tautologischen Äußerungen in unterschiedlichen Sprachen an; z. B. kann das englische:

(25) boys are boys

im Französischen so nicht ausgedrückt werden, vgl.:

(26*) les garçons sont les garçons

(vgl. aber: les garçons ce sont les garçons) 
Die zweite These, die Wierzbicka aufstellt, ist die, daß die sprachliche Form die Bedeutung der tautologischen Äußerungen determiniert; unter sprachlicher Form versteht sie einmal die syntaktische Form der Nominalphrasen und zum andern die Kategorie des Nominalausdrucks. Insgesamt gibt es für das Englische die folgenden Möglichkeiten:

$\mathrm{N}_{\mathrm{abstr}}$ is $\mathrm{N}_{\mathrm{abstr}}$

$\mathrm{N}_{\mathrm{pl}}$ are $\mathrm{N}_{\mathrm{pl}}$

An $\mathrm{N}$ is an $\mathrm{N}$

The $\mathrm{N}$ is the $\mathrm{N}$

$N_{1}$ is $N_{1}$ (and $N_{2}$ is $N_{2}$ ) war is war

kids are kids

a party is a party

the law is the law

Tautologische Äußerungen der ersten Form wie war is war drücken eine vernünftige Einstellung zu komplexen menschlichen Aktivitäten aus, speziell zu solchen, die negative Konsequenzen haben. Im einzelnen sieht die Beschreibung der Bedeutung dieser Untergruppe tautologischer Äußerungen folgendermaßen aus:

A: Jeder weiß, wenn Leute Dinge dieser Art tun, daß diese negative Konsequenzen für andere Leute haben werden

B: Sprecher (S) nimmt an, daß er nicht sagen muß, welche Dinge das sind

C: Wenn man sieht, daß solche negativen Dinge passieren, sollte man sich nicht veranlaßt sehen, etwas Schlechtes zu empfinden

D: Man sollte verstehen, daß es nicht anders sein kann/man es nicht ändern kann

Für die zweite Subgruppe von tautologischen Äußerungen der Form boys are boys, women are women etc. gilt, daß mit ihnen Toleranz für die menschliche Natur ausgedrückt werden soll. Die explizite Bedeutungsformel ist ähnlich wie (28):

(29)

A: Jeder wei $\beta$, daß Leute dieser Art einige Dinge tun, von denen man nicht will, da $\beta$ Leute sie tun

B: S nimmt an, daß S nicht sagen braucht, welche Dinge das sind

C: Wenn jemand sieht, daß Leute dieser Art solche Dinge tun, sollte man sich nicht veranlaßt sehen, etwas Schlechtes zu fühlen

D: Man sollte verstehen, daß Leute dieser Art nicht von selbst Dinge in der Weise tun, wie sie andere Leute wollen

E: Das kann nicht geändert werden

In einer dritten Gruppe werden die letzten drei Fälle mit Artikeln zusammengefaßt; mit ihnen werden Obligationen ausgedrückt. Beispiele sind:

(30) A rule is a rule

(31) A bet is a bet

(32) A promise is a promise

(33) A deal is a deal

(34) The law is the law

(35) A husband is a husband 
Die Bedeutungsformel lautet:

(36)

A: Jeder weiß, daß es bestimmte Dinge gibt, die man $X$ gegenüber tun muß

B: Jeder weiß, daß man manchmal diese Dinge nicht tun will

C: Man muß verstehen, $d a ß$ wir sie tun müssen

D: Man soll sich nicht veranlaßt sehen, etwas Schlechtes darüber zu empfinden

E: Man sollte verstehen, daß dies nicht geändert werden kann

Die semantische Lösung von Wierzbicka hat m. E. drei gravierende Mängel:

(1) Das Argument der unterschiedlichen sprachlichen Formen in verschiedenen Sprachen ist nicht stichhaltig, weil es von falschen Voraussetzungen ausgeht: Sprachen sind prinzipiell nicht ohne formale Transformationen ineinander übersetzbar. Dies schließt aber nicht aus, daß Äußerungen mit den gleichen propositionalen Gehalten (mit gleichen konzeptuellen Strukturen) nicht mit übereinzelsprachlichen Prinzipien beschrieben werden könnten;

(2) Die einzelnen Bedeutungskomponenten, in denen Sprechereinstellungen thematisiert sind, erscheinen mir, was ihren substantiellen Gehalt angeht, ziemlich ad-hocistisch. Aber was schwerwiegender ist: Die Einstellungskomponente der Bedeutung der beiden ersten Subgruppen: „S nimmt an, daß er nicht zu sagen braucht, welche Dinge das sind" scheint mir aus dem Rahmen zu fallen, da sie auf die kommunikative Situation der tautologischen Äußerung rekurriert. Sie besagt ja auch, daß der Sprecher seinen Adressaten so einschätzt, daß er jeweils weiß, welche Dinge dieser weiß, d. h. auch, daß mit dieser Einstellung letztlich auf das Prinzip der Relevanz Bezug genommen wird, und dies ist ganz klar ein universalpragmatisches Prinzip!

(3) Und das ist vielleicht das Schlimmste: die einzelnen Analysen stimmen einfach empirisch nicht: Ein Text aus der ZEIT soll dies belegen:

(37)

Wir glauben jedoch nicht, daß es einmal eine Menge wissenschaftlicher Prinzipien für die ganze Welt geben soll und eine andere Menge für Experimente mit Zigaretten. Wissenschaft ist Wissenschaft. Beweis ist Beweis. Daher bleibt die Auseinandersetzung über Rauchen und Gesundheit offen.

(ZEIT, 13. 1. 1988.)

Weder der Ausdruck Wissenschaft noch der Ausdruck Beweis bezeichnen komplexe Aktivitäten mit negativen Konsequenzen. Eine tautologische Äußerung wie: Asylanten sind Asylanten scheint mir in allen Kontexten auch nicht unbedingt zur Toleranz aufzurufen, oder gar: Ausländer sind Ausländer!

Wir sind jetzt an dem Punkt angekommen, an dem wir zugeben müssen: die semantischen Ansätze sind in unterschiedlicher Weise gescheitert. Der Ansatz, tautologische Äußerungen als Spezialfall von Identitätssätzen zu betrachten, brachte eine Unzahl von Bedeutungsvermehrungen mit sich, und der Ansatz der Einstellungssemantik war, abgesehen von der empirischen Inadäquatheit, nicht frei von pragmatischen Aspekten. Wenden wir uns jetzt dem genuin pragmatischen Ansatz zu. 


\section{Radikal pragmatische Lösungen}

Bereits Levinson plädiert in seinem Buch „Pragmatics“ (1983) für einen radikal pragmatischen Ansatz zur Erklärung der Informativität tautologischer ÄuBerungen. Er schlägt vor, ihren semantischen Beitrag als vernachlässigbar zu betrachten und die Äußerungsinterpretation vollständig der pragmatischen Inferenz im Griceschen Sinn zuzuschreiben: ,an account of how tautologies come to have communicative significance, and different communicative significances, can be given in terms of the flouting of the maxim of Quantity. Since this requires that speakers be informative, the asserting of tautologies blatantly violates it. Therefore, if the assumption that the speaker is actually cooperating is to be preserved, some informative inference must be made." (Levinson, 1983, 111) Die jeweils nicht-konventionell implizierte (oder implikatierte) Information hängt in hohem Maß, wenn nicht gänzlich, von dem Kontext der Äußerung ab. Um verstanden zu werden, muß der Sprecher also davon ausgehen können, daß sein Adressat das „Offenkundige“ der Information zu erkennen in der Lage ist (vgl. Rolf, 1994). Der Ansatz von Levinson ist insofern radikal pragmatisch, als mit ihm lediglich erfaßt wird, daß mit tautologischen Äußerungen Informationen mitgeteilt werden, aber nicht, welche Informationen dies sind. Eine weniger radikale Lösung schlägt Fraser (1988) vor, der davon ausgeht, daß mehr konventionelle Bedeutung mit tautologischen Konstruktionen verknüpft ist. Seine Bestimmung:

(38)

Mit einer tautologischen Äußerung gibt ein Sprecher zu verstehen, daß er beabsichtigt, $\mathrm{da} ß$ der Adressat erkennt:

(i) daß der Sprecher eine spezielle Sichtweise gegenüber den Objekten, auf die mit der NP referiert wird, einnimmt;

(ii) daß der Sprecher glaubt, daß der Hörer diese spezielle Sichtweise erkennen kann;

(iii) $\mathrm{da} B$ diese Sichtweise für die Kommunikation relevant ist.

(Fraser, 1988, 217/218.)

In dieser Bestimmung ist nicht spezifiziert, welche Sichtweise ein Sprecher jeweils einnimmt; vielmehr wird lediglich betont, daß ein Sprecher mit einer tautologischen Äußerung einen bestimmten Glauben zu verstehen zu geben beabsichtigt, nämlich den Glauben, daß die Gesprächsteilnehmer (speziell der jeweilige Adressat) eine spezielle Sichtweise auf den Referenten der NP mit ihm teilen. Die illokutionäre Kraft einer solchen Äußerung ist dieselbe wie die einer Feststellung: der Sprecher beabsichtigt, diesen Glauben in das Bewußtsein des Adressaten zu bringen. Welche spezielle Sichtweise jeweils eingenommen wird, ist Sache des Kontextes einer Äußerung.

In einer Replik auf die semantische Analyse von Wierzbicka und auf die ihrer Meinung nach zu vagen Versuche einiger Griceaner haben Ward und Hirschberg (1991) eine Neuinterpretation von tautologischen Äußerungen versucht. Ausgangspunkt ist zunächst eine Einteilung der Formen tautologischer Äußerungen: 
(39)

Equitative:

Disjunktionen:

Konditionale:

Relative:

a ist a Krieg ist Krieg, Kinder sind Kinder

(entweder) $\mathrm{p}$ oder nicht $\mathrm{p}$

wenn $p$, dann $p$

$p$, was $p$

Die Interpretation einer tautologischen Aussage einer dieser Formen kann folgendermaßen beschrieben werden:

(40)

Angenommen ein Sprecher S hat eine tautologische Aussage einem Hörer $\mathrm{H}$ gegenüber geäußert, etwa der Art von Krieg ist Krieg, dann kann $\mathrm{H}$ folgendermaßen räsonnieren:

(i) $\mathrm{S}$ hat eine tautologische Aussage der Form $a$ ist $a$ geäußert, die nichts zu unserem gegenseitigen Wissen im allgemeinen und nichts zu unserem besonderen Wissen über a hinzufügt;

(ii) Es gibt keinen Grund anzunehmen, daß S gegen das Kooperationsprinzip verstößt, und aufgrund der Maximen der Qualität und der Relevanz hat S soviel über a gesagt, was er wahrheitsgemäß sagen kann;

(iii) $\mathrm{S}$ hätte auch eine Aussage der ähnlichen Form, etwa „a ist b“, machen können, wo $a$ und $b$ verschiedene referierende Ausdrücke sind, was etwas zu unserem gegenseitigen Wissen über a hinzugefügt hätte;

(iv) S hat solche Alternativen nicht gewählt;

(v) daher impliziert $\mathrm{S}$ (nicht konventionell), daß diese Alternativen nicht relevant für die Zwecke unserer Konversation sind.

Das heißt: tautologische Äußerungen markieren, daß es in diesem besonderen Konversationszusammenhang nicht auf spezielle Charakterisierungen, dies wären die Alternativen, ankommt, daß diese nicht gefragt, daß sie irrelevant sind, was auf alle tautologischen Äußerungen in gleicher Weise zutreffen soll. Irrelevant sind die speziellen Charakterisierungen in ihrer Eigenschaft als Informationen über die Beschaffenheit eines Gegenstands, auf den in der ersten Nominalphrase tautologischer Äußerungen Bezug genommen wird, deswegen, weil sie in der entsprechenden Äußerungssituation vom jeweiligen Sprecher als Elemente des mit dem Hörer geteilten Gegenstandswissens (voraus)gesetzt werden: Über Sachverhalte, die man als vom Adressaten gekannt und für wahr gehalten zugleich einschätzt, spricht man nicht, es sei denn, man will den Befund des geteilten Wissens für sich selbst und den/die Adressaten in einer bestimmten Situation in Erinnerung bringen oder besser und im wahrsten Sinn des Wortes: ,vergegenwärtigen'. Tautologische Äußerungen, so könnte man also auch sagen, haben eine spezifische metakommunikative Funktion. Feilke, der Tautologien und Truismen als „Evidenzformeln“ zusammenfaßt, spricht von einer „,kommunikationssemantische[n], die phatische Gemeinschaft ,konfirmierende[n]' Funktion “ (Feilke, 1996, 309). Miki (1996) bestimmt Tautologien als Formen der Selbstidentifikation derart, daß ein Sprecher sich auf geteiltes Wissen bezieht und dieses durch „evocation“, d. h. eine Art Appell in das Bewußtsein des Adressaten bringt: „this whole process may be viewed as a reaffirmation or reestablishment of shared beliefs" (Miki, 1996, 641). Diese Analysen korrespondieren mit einigen Befunden aus der Gesprächsanalyse. Gülich (1981) und Quasthoff (1978) haben zum Beispiel festgestellt, daß clichehafte Formeln, Routineformeln, Alltagsweis- 
heiten, Truismen u. ä., wozu als Spezialfall auch tautologische Äußerungen gehören, dazu dienen, Komplexität der thematischen Behandlung in der Kommunikation zu reduzieren. Drei authentische Beispiele sollen im folgenden belegen, da $ß$ die pragmatische Analyse sowie die konversationsanalytische Beobachtung zutreffend sind:

(41)

(Die Rede ist hier von einem Spediteur, der einen Freiballon abtransportiert in der Annahme, es sei ein Bienenkorb.)

Als er später gefragt wurde, was er sich denn dabei eigentlich gedacht habe und ob ihm nicht aufgefallen sei, daß es kein Bienenkorb war, entgegnete der junge Mann nur: „Na, ja - ich hab mir wohl so was gedacht, aber Auftrag ist Auftrag!"

(Beispiel aus Gülich, 1981, 360)

(42)

Alle Behörden, mit denen Erika de Crouppe zu tun hatte, berufen sich auf die Buchstaben des Gesetzes. Ein Härtefall, schreibt das Arbeitsamt in einem Brief, sei Erika de Crouppe sicher, aber Gesetz sei eben Gesetz. Zugegeben: Gesetze können nicht für jeden schwierigen Einzelfall Lösungen anbieten. Aber es gibt den Ermessensspielraum - den Raum für menschliche Entscheidungen. Nach dem hat aber keiner der Sachbearbeiter für Erika de Crouppe suchen wollen.

(Beispiel aus Gülich, 1981, 361)

(43)

Die Dokumente zeigen, daß alle diese Balanceakte eines majestätisch auftretenden, doch inmitten der Weltkatastrophe tief verunsicherten Papstes nicht so schematisch gedeutet werden können, wie es bei der Hochhuth-Debatte bei Gegnern und Bewunderern des zwölften Pius oft geschah. „Man tut, was man tut" lautet die immer wiederkehrende Formel. Ob ein Papst mehr tun sollte, ist jedoch eine Frage des moralischen, nicht des historisch-politischen Ermessens.

(ZEIT, 13. 12. 1976)

In all diesen Texten wird deutlich, daß in dem jeweils geschilderten Zusammenhang kein Spezialurteil über den jeweils thematisierten Sachverhalt relevant ist: im ersten Beispiel (36) wird durch die adversative Konjunktion aber signalisiert, daß spezielle Überlegungen zur Aufgabe des Spediteurs keine Rolle spielten. In Beispiel (37) signalisiert aber in gleicher Weise, daß es keine besonderen Alternativen gibt, und in Beispiel (38) wird durch die tautologische Äußerung jegliche substantielle Argumentation abgeblockt.

\section{Ausblick}

$\mathrm{Zu}$ Beginn dieses Beitrags habe ich auf den von Grice präzisierten Unterschied zwischen Konventionalität von Sprache und Kooperativität von Sprechern hingewiesen. Bezüglich der diskutierten Fälle tautologischer Äußerungen könnte man nun einwenden, daß ihre Verwendung nicht ohne sprachlich determinierte Konventionen auskommt, insofern sie an ganz bestimmte syntaktische Muster gebunden sind oder, wie Feilke (1996) sagt, bestimmte „Prägungen“ darstellen, die konventionalisierte Deutungsmuster auslösen. Das gilt, wie auch Wierzbicka betont hat, in zweierlei Hinsichten: 
- einmal gibt es in den jeweiligen Einzelsprachen feste syntaktische Muster für tautologische Äußerungen;

- zum anderen variieren die syntaktischen Muster von Sprache zu Sprache.

Andererseits legen die konventionell ausgelösten Deutungsprozesse keine invarianten Interpretationen über alle Situationen hinweg fest. Was in einer bestimmten Äußerungssituation als gemeinsames Gegenstandswissen zu gelten hat, bleibt den kooperativen Anstrengungen der Kommunikationsteilnehmer überlassen, und diese begründen - im Rahmen der vorgegebenen einzelsprachlichen Konventionen - partielle situationsabhängige Wissens- und Kommunikationsgemeinschaften: Dadurch, daß Sprecher nicht-konventionelle Festlegungen treffen, schaffen sie Meinens- und Verstehensfundamente, die innerhalb einer Gruppe spezifische Gemeinschaften mit jeweiligen Überzeugungssystemen in einer bestimmten Situation begründen können. Dies gilt für all die Fälle, in denen etwas anderes gesagt wird als zu verstehen gegeben werden soll. Einen dieser Fälle stellen tautologische Äußerungen dar, mit denen an eine Gemeinschaft von „Wissenden“ appelliert wird, wobei die Art des Wissens als für den jeweiligen Zusammenhang irrelevant unterstellt ist: „man versteht sich eben" auch ohne lange Erklärungen oder linguistisch: Charakterisierungen.

\section{Literatur}

Feilke, Helmuth (1996): Sprache als soziale Gestalt. Ausdruck, Prägung und die Ordnung der sprachlichen Typik. Frankfurt/M.: Suhrkamp.

Fraser, Bruce (1988): Motor oil is motor oil. In: Journal of Pragmatics 12, 1988, 214-220.

Grice, Paul (1989): Studies in the Way of Words. Cambridge/Mass./London: Harvard University Press.

Gülich, Elisabeth (1981): „Was sein muß, muß sein“. Überlegungen zum Gemeinplatz und seiner Verwendung. In: Weydt, H. (Hrsg.): Logos Semantikos. Vol II: Sprachtheorie und Sprachphilosophie. Berlin/Madrid: de Gruyter/Editorial Gredos.

Levinson, Stephen C. (1983): Pragmatics. Cambridge: Cambridge University Press.

Miki, Etsuzo (1996): Evocations and tautologies. In: Journal of Pragmatics 25, $635-648$.

Quasthoff, Uta (1978): The Uses of Stereotypes in Everyday Argument. In: Journal of Pragmatics 2, 1978, 1-48.

Rolf, Eckard (1994): Sagen und Meinen. Paul Grices Theorie der Konversations-Implikaturen. Opladen: Westdeutscher Verlag.

Tugendhat, Ernst (1976): Vorlesungen zur Einführung in die sprachanalytische Philosophie. Frankfurt/M.: Suhrkamp.

Tugendhat, Ernst \& Ursula Wolf (1983): Logisch-semantische Propädeutik. Stuttgart: Reclam.

Ward, Gregory L. \& Julia Hirschberg (1991): A pragmatic analysis of tautological utterances. In: Journal of Pragmatics 15, 1991, 507-520.

Wierzbicka, Anna (1987): Boys will be boys. ,Radical Semantics‘ vs. ,Radical Pragmatics'. In: Language 63, 1987, 95-114.

Wierzbicka, Anna (1988): Boys will be Boys. A rejoinder to Bruce Fraser. In: Journal of Pragmatics 12, 1988, 221-224.

Adresse der Verfasserin:

Prof. Dr. Gisela Harras, Institut für deutsche Sprache, R 5, 6-13, D-68161 Mannheim 